\title{
Intratumoral estrogen sulfotransferase induction contributes to the anti-breast cancer effects of the dithiocarbamate derivative TM208
}

\author{
Xi-wei $\mathrm{JI}^{1,2}$, Guang-ping $\mathrm{CHEN}^{4}$, Yan SONG ${ }^{5}$, Ming HUA ${ }^{1}$, Li-jie WANG ${ }^{1}$, Liang $\mathrm{LI}^{1}$, Yin YUAN ${ }^{1}$, Si-yuan WANG ${ }^{1}$, \\ Tian-yan $\mathrm{ZHOU}^{1,3, *}$, Wei $\mathrm{LU}^{1,3}$ \\ ${ }^{1}$ Department of Pharmaceutics, School of Pharmaceutical Sciences, Peking University, Beijing 100191, China; ${ }^{2}$ Institute of Clinical \\ Pharmacology, Peking University First Hospital, Beijing 100191, China; ${ }^{3}$ State Key Laboratory of Natural and Biomimetic Drugs, Peking \\ University, Beijing 100191, China; ${ }^{4}$ Department of Physiological Sciences, Center for Veterinary Health Sciences, Oklahoma State \\ University, Stillwater, OK 74078, USA; ${ }^{5}$ Department of Molecular and Cellular Pharmacology, School of Pharmaceutical Sciences, \\ Peking University, Beijing 100191, China
}

\begin{abstract}
Aim: Sulfotransferase-catalyzed sulfation is the most important pathway for inactivating estrogens. Thus, activation of estrogen sulfotransferase (EST) may be an alternative approach for the treatment of estrogen-dependent breast cancer. In this study we investigated the involvement of EST in anti-breast cancer effects of the dithiocarbamate derivative TM208 in vitro and in vivo. Methods: The viability of human breast cancer MCF-7 cells was determined using a SBB assay. Nude mice bearing MCF-7 cells were orally administered TM208 (50 and $\left.150 \mathrm{mg} \cdot \mathrm{kg}^{-1} \cdot \mathrm{d}^{-1}\right)$ for 18 days. The xenograft tumors and uteri were collected. The mRNA expression of EST was examined with real-time PCR. EST protein was detected with Western blot, ELISA or immunohistochemical staining assays. A radioactive assay was used to measure the EST activity. Uterotropic bioassay was used to examine the uterine estrogen responses. Results: Treatment with TM208 (10, 15 and 20 mol/L) concentration-dependently increased EST expression in MCF-7 cells in vitro. Co-treatment with triclosan, an inhibitor of sulfonation, abolished TM208-induced cytotoxicity in MCF-7 cells. TM208 exhibited an apparent anti-estrogenic property: it exerted more potent cytotoxicity in E2-treated MCF-7 cells. In the nude mice bearing MCF-7 cells, TM208 administration time-dependently increased the expression and activity of EST, and blocked the gradual increase of E2 concentration in the xenograft tumors. Furthermore, TM208 administration blocked the estrogens-stimulated uterine enlargement. Tamoxifen, a positive control drug, produced similar effects on the expression and activity of EST in vitro and in vivo.

Conclusion: The induction of EST and reduction of estrogen concentration contribute to the anti-breast cancer action of TM208 and tamoxifen. TM208 may be developed as anticancer drug for the treatment of estrogen receptor-positive breast cancer.
\end{abstract}

Keywords: breast cancer; dithiocarbamate; tamoxifen; estrogen; estrogen sulfotransferase; human tumor xenograft model; uterotropic bioassay

Acta Pharmacologica Sinica (2015) 36: 1246-1255; doi: 10.1038/aps.2015.14; published online 4 May 2015

\section{Introduction}

Most breast cancers are estrogen-dependent and require the presence of estrogens to maintain growth during their development, and two-thirds of breast cancers occur during the postmenopausal period when the ovaries are no longer functional. Therefore, intratumoral estrogens, especially $17 \beta$-estradiol (E2), play pivotal roles in the proliferation and development of hormone-dependent breast carcinoma cells ${ }^{[1-3]}$. Previous studies have demonstrated that the tissue concentra-

\footnotetext{
* To whom correspondence should be addressed.

E-mail tianyanzhou@bjmu.edu.cn

Received 2014-12-15 Accepted 2015-03-02
}

tions of E2 in breast carcinomas are much higher than those found in the plasma ${ }^{[4,5]}$ or normal mammary tissues ${ }^{[6,7]}$. Consequently, blocking in situ estrogen production appears to be a potent breast cancer treatment ${ }^{[8-11]}$. For example, the prevention of estrogen binding by anti-estrogens [such as tamoxifen (Tam) $]^{[12]}$ and the inhibition of estrogen synthesis by aromatase inhibitors (such as Letrozole) have been reported to be effective in clinical treatment ${ }^{[13]}$.

Regulation of the intratumoral estrogen-metabolizing enzyme is another option for decreasing the active estrogen level in tumors. The sulfation of estrogens by sulfotransferase (SULT) is the most important pathway for inactivating estrogens, including estrone (E1) and 17 $\beta$-estradiol (E2). The 
main SULT isoforms that catalyze the sulfation of estrogen in the human body include SULT1E1, SULT1A1, SULT2A1, and SULT2B1, as well as others ${ }^{[8,9,11,14]}$. Among these isoforms, estrogen sulfotransferase (EST, SULT1E1) plays the most important role in estrogen inactivation under physiological conditions due to its high affinity for estrogens ${ }^{[15]}$. In most previous studies, the intratumoral estrogen concentrations were expressed as pg/g tissue or $\mathrm{pmol} / \mathrm{g}$ tissue, which can be approximately calculated at the "nanomolar" (physiological) level even if they are much higher than those found in normal mammary tissues ${ }^{[6,7]}$ or plasma ${ }^{[4,5]}$. Therefore, the intratumoral sulfation of estrogen is mainly catalyzed by EST rather than other SULT isoforms. Moreover, SULT1E1 expression was reported in approximately $44 \%$ of human breast cancer biopsy specimens, and the mRNA expression and enzyme activity of EST are inversely correlated with the breast tumor size and significantly associated with a decreased risk of recurrence ${ }^{[11,16]}$. Therefore, targeted activation of EST is likely to pave an alternative route for success in hormone-dependent breast cancer treatment. Some groups have applied the upregulation of EST by genetic methods ${ }^{[17,18]}$ or through the administration of progestins ${ }^{[19]}$ to treat breast cancer. Some commonly used anticancer drugs (such as Tam) ${ }^{[20]}$ and methotrexate $^{[21]}$ and some natural flavonoid compounds (such as biochanin A and genistein ${ }^{[22]}$ that have been shown to possess potential anti-breast cancer properties have been found to significantly induce rSULT1A1 and rSULT2A1 in the livers of normal rats. However, to the best of our knowledge, few studies have examined the endocrine therapy of breast cancer via intratumoral EST induction using a candidate anticancer compound.

Dithiocarbamates can prevent or treat a variety of cancers ${ }^{[23,24]}$, and a series of new dithiocarbamate derivatives have been developed, including TM208 (Figure 1$)^{[25,26]}$. TM208 is a promising anticancer candidate because it significantly inhibits the growth of hepatocellular carcinoma, gastric carcinoma, and breast tumors in nude mice, and it has a very low toxicity and good pharmacokinetic characteristics ${ }^{[27-29]}$. TM208 was found to have anti-breast cancer activity in both MCF-7 cells and MCF-7 tumor-bearing mice; it also has an inhibitory effect on the phosphorylation of epidermal growth factor receptor (EGFR), which may play an important role in its anticancer efficacy ${ }^{[29]}$. However, considering that MCF-7 cells express estrogen receptor (ER) with a low level of EGFR expression ${ }^{[30]}$ and that the tumor growth in MCF-7 xenograft mice is sensitive to the intratumoral estrogen concentration, we speculate that TM208 acts via a down-regulation of the in situ estrogen

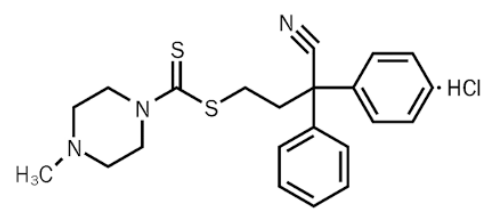

Figure 1. The chemical structure of TM208. concentration in breast carcinoma.

Therefore, the purpose of this study was to investigate the effects of TM208, a chemical compound with anticancer potential, on EST and estrogen in vitro and in vivo and, more importantly, to better understand the role of intratumoral EST induction in breast cancer treatment with TM208.

\section{Materials and methods \\ Drugs and reagents}

TM208 (>99.5\%) was provided by Prof Run-tao LI (Peking University $)^{[31]}$. Tam was purchased from Lanospharma Laboratories Co, Ltd (Chongqing, China). RPMI-1640 medium was obtained from Macgene Biotech Co, Ltd (Beijing, China), and fetal bovine serum was purchased from Gibco (Grand Island, USA). Triclosan was purchased from Merck (Darmstadt, Germany). Rabbit anti-human EST antibody (for the Western blot assay) ${ }^{[32]}$ was a gift from Dr David RINGER (American Cancer Society $)^{[21]}$. The peptide antigen sequence used for generating this antibody is $\mathrm{NH}_{2}$-(Glu-Gln-Gln-Met-Lys-Glu-SerThr-Leu-Lys-Phe-Arg-Thr-Glu-ILe ${ }^{[33]}$. The specificity of this antibody has been confirmed in Dr Ringer's laboratory. Rabbit anti-human actin antibodies were purchased from Beijing Biosynthesis Biotechnology Co, Ltd (Beijing, China). Horseradish peroxidase-conjugated anti-rabbit antibodies were purchased from Cell Signaling Technology (Danvers, MA, USA). ${ }^{3} \mathrm{H}$-estradiol was obtained from PerkinElmer, Inc (Boston, MA, USA). Rabbit anti-EST antibody (for immunohistochemical staining) and 3'-phosphoadenosine-5' -phosphosulfate (PAPS) were purchased from Sigma-Aldrich (St Louis, MO, USA).

\section{Cell culture}

The MCF-7 cell line was purchased from the Institute of Materia Medica Academy of Medical Sciences (China). MCF-7 cells were cultured in RPMI-1640 medium supplemented with 10\% fetal bovine serum, $100 \mathrm{U} / \mathrm{mL}$ of penicillin and $100 \mu \mathrm{g} / \mathrm{mL}$ of streptomycin. These cells were maintained at $37^{\circ} \mathrm{C}$ in a mixture of $5 \% \mathrm{CO}_{2}$ and $95 \%$ air.

\section{Sulforhodamine B assay on the sulfation-inhibitory effects of triclosan}

The sulforhodamine B (SRB) assay is typically used to detect the survival rate of cells treated with cytotoxic compounds ${ }^{[29]}$. Here, it was further applied to investigate the effects of triclosan, an inhibitor of sulfation, on the survival rate of MCF-7 cells exposed to TM208. Briefly, MCF-7 cells growing at the exponential phase were seeded into 96-well microplates at a density of $1 \times 10^{4}$ cells/well. After $24 \mathrm{~h}$ of incubation, the cells were treated for $48 \mathrm{~h}$ with vehicle, TM208 $(20 \mu \mathrm{mol} / \mathrm{L}$, diluted in DMSO) and triclosan $(0,0.1$, and $0.2 \mu \mathrm{mol} / \mathrm{L}$, diluted in DMSO), with 6 replicate wells for each triclosan concentration. Then, detection followed a published protocol ${ }^{[34]}$. The percentage of viable cells in response to TM208 with different concentrations of triclosan was calculated using the following formula: Percentage of cell survival $=O D_{\text {Triclosan\&TM208 }} /$ $O D_{\mathrm{TM} 208} \times 100 \%$. 
Sulforhodamine B assay on the antiestrogenic activity of TM208 The cell viability was determined using the SRB assay as described above. To evaluate the antiestrogenic activity of TM208, we first evaluated the effects of E2 on the viability of MCF-7 cells. MCF-7 cells were cultured in phenol red-free and hormone-free RPMI-1640 solvents with different concentrations of E2 (0.001 nmol/L, $1 \mathrm{nmol} / \mathrm{L}, 1 \mu \mathrm{mol} / \mathrm{L}$, and 100 $\mu \mathrm{mol} / \mathrm{L}$; diluted in DMSO), and the control group was treated with DMSO. The percentage of cell survival rate $=O D_{\mathrm{E} 2} /$ $O D_{\text {control }} \times 100 \%$. Then, MCF-7 cells were cultured in RPMI1640 media mixed with $1 \mathrm{nmol} / \mathrm{L} \mathrm{E}^{[35]}$, and different concentrations of TM208 or Tam were added. The corresponding cell survival rates were determined, and the value of the $\mathrm{IC}_{50}$ of TM208 or Tam was calculated.

\section{Animals}

Beijing Vital Laboratory Animal Technology (Beijing, China) provided female BALB/c nude mice (5-6 weeks old). These nude mice were housed in an animal room under controlled conditions (temperature $22-24^{\circ} \mathrm{C}$, humidity $55 \%-60 \%, 12 \mathrm{~h}$ light/12 $\mathrm{h}$ dark cycle) and had free access to food and water. This research adhered to the Principles of Laboratory Animal Care (NIH publication No 85-23, revised 1996). All experiments were performed under the approval of the Experiment Administration Committee of the Peking University Health Science Center.

\section{Tumor xenograft model}

MCF-7 cells $\left(2 \times 10^{6}\right)$ were suspended in $200 \mu \mathrm{L}$ of PBS (pH 7.4) and then subcutaneously inoculated into both second mammary fat pads of 5-week-old virgin female BALB/c nude mice. The tumor diameter was measured with a vernier caliper and converted to tumor volume using the formula $1 / 2 \times A \times B^{2}$ ( $\mathrm{A}=$ larger diameter, $\mathrm{B}=$ smaller diameter). Treatment was started when the average tumor volumes reached $150 \mathrm{~mm}^{3}$.

\section{Tumor growth inhibition assay}

Xenograft mice were randomly divided into four groups with four mice per group. TM208 was dissolved in 10\% w/v hydroxypropyl- $\beta$-cyclodextrin aqueous solution and administered by gavage at 50 and $150 \mathrm{mg} \cdot \mathrm{kg}^{-1} \cdot \mathrm{d}^{-1}$. The blank control group received only vehicle solution, whereas the positive control group received $50 \mathrm{mg} \cdot \mathrm{kg}^{-1} \cdot \mathrm{d}^{-1}$ Tam solution. The tumor size was measured every third day. After $18 \mathrm{~d}$ of treatment, animals were euthanized by cervical displacement. Tumors and uteruses were collected after the final treatment and frozen at $-80^{\circ} \mathrm{C}$ until use.

\section{Western blot analysis}

In vitro, MCF-7 cells were treated with TM208 or Tam for $7 \mathrm{~d}$. The positive control consisted of MCF-7 cells incubated with Tam for $7 \mathrm{~d}$. Then, the cells were harvested, and cytosol was prepared according to a previous study ${ }^{[21]}$. In vivo, tumor-bearing mice were treated with TM208 for $18 \mathrm{~d}$, and cytosol samples from the tumors were prepared as previously described $^{[36]}$.
Western blot analysis was conducted as described in a previous study ${ }^{[36]}$. Total protein $(50 \mu \mathrm{g})$ was separated by SDS$12 \% w / v$ polyacrylamide gel electrophoresis and transferred to PVDF membranes (Pierce, Rockford, IL, USA). The dilution ratio of primary antibody was 1:1000. Horseradish peroxidase-linked anti-rabbit antibody was used as the secondary antibody. The membrane was developed with Supersignal Ultra (Pierce, Rockford, IL, USA). Individual protein bands were quantified by densitometry using the Image system (BioRad Laboratories, Hercules, CA, USA) and normalized for $\beta$-actin.

\section{Quantitative real-time PCR}

In vitro, MCF-7 cells were prepared as described above. In vivo, the MCF-7 xenograft mice were randomly divided into two groups and treated with vehicle solution and $150 \mathrm{mg} / \mathrm{kg}$ TM208, respectively. Tumors were obtained from three mice per group at $0,3,6,9$, and $12 \mathrm{~d}$. The total RNA was extracted using an RNA isolation kit (Takara, Shiga, Japan) according to the manufacturer's instructions. cDNA was synthesized from $2 \mu \mathrm{g}$ of RNA using a reverse transcriptase M-MLV synthesis kit (Takara). Real-time PCR was performed using the MyiQ5 real-time PCR detection system (Bio-Rad) with SYBR Premix Ex Taq (Takara). The PCR products for human $\beta$-actin and EST were synthesized with the primer pairs FP (forward primer) 5'-CGCGAGAAGATGACCCAGAT-3' and RP (reverse primer) 5'-TCACCGGAGTCCATCACGAT-3' (hACTIN, Gene ID: 60) as well as FP 5'-ATCTTGTCATTGCCACCTAC-3' and RP 5'-TGGCAAATGAGTCTTCAC-3' (EST, Gene ID: 6783). All primers were purchased from AuGCT DNA-SYN Biotechnology Co, Ltd (Beijing, China). Samples were run in triplicate under the following conditions: an initial denaturation for $30 \mathrm{~s}$ at $95^{\circ} \mathrm{C}$, followed by 45 cycles $^{[37]}$ of $15 \mathrm{~s}$ at $95^{\circ} \mathrm{C}, 30 \mathrm{~s}$ at $60^{\circ} \mathrm{C}$, and $33 \mathrm{~s}$ at $72^{\circ} \mathrm{C}$. The levels of gene expression in each sample were normalized to $\beta$-actin mRNA.

\section{Radioactive assay on the activity of EST}

Tumor-bearing mice were treated with TM208 for $18 \mathrm{~d}$, and the cytosol of the tumors was prepared as previously described $^{[36]}$. The reaction mixture, containing $0.15 \mu \mathrm{mol} / \mathrm{L}$ ${ }^{3} \mathrm{H}$-estradiol as the substrate ${ }^{[38]}, 50 \mathrm{mmol} / \mathrm{L}$ Tris buffer $(\mathrm{pH}$ 6.2), $20 \mu \mathrm{mol} / \mathrm{L}$ PAPS, and $20 \mu \mathrm{L}$ of cytosol, as well as 100 $\mu \mathrm{g}$ of total protein as the enzyme source, in a total volume of $250 \mu \mathrm{L}$, was incubated at $37.0^{\circ} \mathrm{C}$ for $30 \mathrm{~min}$. The reaction was stopped with the addition of $250 \mu \mathrm{L}$ of $0.25 \mathrm{~mol} / \mathrm{L}$ Tris $(\mathrm{pH}$ 8.7) and $1 \mathrm{~mL}$ of chloroform for extraction. The extraction was repeated twice. An aliquot $(100 \mu \mathrm{L})$ of the water phase was used for scintillation counting with a Tri-carb 2100TR scintillation counter (PerkinElmer Life Sciences, Inc, Waltham, MA, USA). For controls, PAPS was eliminated. Assays were performed in triplicate, and the average of the measurements minus the controls was used to calculate the enzymatic activity.

\section{Immunohistochemical staining}

Paraffin sections of the tumors were used for Immunohisto- 
chemical (IHC) detection. IHC was performed according to the published protocol ${ }^{[39]}$. The slides were incubated with a 1:40 dilution of the primary antibody of EST.

\section{Measurement of tumor estrogen levels}

Xenograft mice were randomly divided into two groups, a vehicle-control group and a continuous-dose group. Tumorbearing mice in the two groups $(n=3)$ were treated with vehicle solution and $150 \mathrm{mg} \cdot \mathrm{kg}^{-1} \cdot \mathrm{d}^{-1} \mathrm{TM} 208$, respectively. Tumors were collected from three mice per group at $0,3,6,9$, and $12 \mathrm{~d}$. These tumors were washed in ice-cold saline and snap-frozen. All tumors were cut into small pieces and sonicated for $25 \mathrm{~s}$ in ice-cold PBS ( $\mathrm{pH}=7.4)$. The homogenates were centrifuged at $12000 \times g$ for $30 \mathrm{~min}$ at $4{ }^{\circ} \mathrm{C}$, and the supernatant was collected. The concentration of free estrogen in cytosol containing $100 \mu \mathrm{g}$ of total protein was measured using the HUMAN ESTROGEN ELISA kit from Puli Zhicheng Biotechnology Co, Ltd (Beijing, China).

\section{Uterotropic bioassay}

Tumor-bearing nude mice were treated with TM208, Tam or vehicle daily for $18 \mathrm{~d}$, as described above. Mice were sacrificed after the last treatment. The harvested uteruses were dissected and weighed.

\section{Statistics}

Data are presented as the mean $\pm \mathrm{SD}$. One-way analysis of variance (ANOVA) was used to determine the significance among the groups and then post hoc tests with the Bonferroni correction were used for multiple comparisons between individual groups. Differences were considered statistically significant when the $P$ value was less than 0.05 . Statistical analyses were performed using GraphPad Prism 5.0 software (GraphPad Software, Inc, USA).

\section{Results}

\section{TM208 inhibited the tumor growth of MCF-7 xenografts}

TM208 can decrease the cell viability of MCF-7 cells, as we have previously described ${ }^{[29]}$. The $\mathrm{IC}_{50}$ values of TM208 and Tam in MCF-7 cells were 36.38 and $22.45 \mu \mathrm{mol} / \mathrm{L}$, respectively. These results indicate that Tam has a stronger effect, associated with the reduced survival of MCF-7 cells, than that of TM208. In the MCF-7 xenograft model, TM208 treatment caused a significant inhibition in tumor growth compared with vehicle treatment (Figure 2A). On d 18, the tumor inhibitory rates of TM208 (50 and $\left.150 \mathrm{mg} \cdot \mathrm{kg}^{-1} \cdot \mathrm{d}^{-1}\right)$ and Tam $\left(50 \mathrm{mg} \cdot \mathrm{kg}^{-1} \cdot \mathrm{d}^{-1}\right)$ were $41.77 \% \pm 5.01 \%, 70.11 \% \pm 5.86 \%$, and $47.41 \% \pm 1.44 \%$, respectively. The inhibition of tumor growth induced by a low dose of TM208 $\left(50 \mathrm{mg} \cdot \mathrm{kg}^{-1} \cdot \mathrm{d}^{-1}\right)$ was similar to that induced by Tam; however, the group treated with a high dose of TM208 $\left(150 \mathrm{mg} \cdot \mathrm{kg}^{-1} \cdot \mathrm{d}^{-1}\right)$ had smaller mean volumes in the tumors compared with those of both the Tam group and the low-dose TM208 group. The body weights of TM208-treated mice in different groups were similar to those of the vehicle-treated mice (Figure 2B). A previous study showed that the $200 \mathrm{mg} / \mathrm{kg}$ dose of TM208 had little effect on
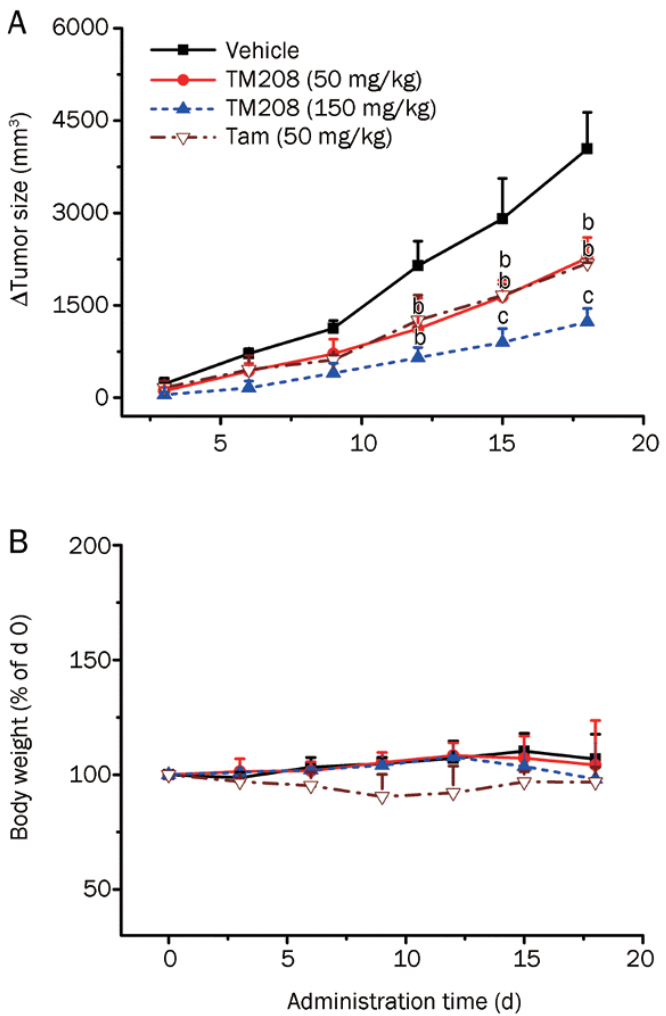

Figure 2. Inhibitory effects of TM208 on MCF-7 xenograft tumors. (A) TM208 treatment caused a significant inhibition in tumor growth compared with vehicle treatment. $\Delta$ Tumor size $=(\text { Tumor size })_{t}-($ Tumor size $)_{d}$. (B) Body weights at different times in the TM208-treated groups were similar to those in the vehicle-treated group, indicating that TM208 has low toxicity. $n=4 .{ }^{b} P<0.05,{ }^{c} P<0.01$ vs vehicle.

leukocytes ${ }^{[27]}$. All of these results suggest that TM208 inhibits the growth of MCF-7 xenograft tumors and has low toxicity.

\section{TM208- and tamoxifen-induced EST expression in MCF-7 cells}

Real-time PCR results showed that the basal mRNA expression of EST in MCF-7 cells is very low, and the mean Ct value is approximately 34.90 (3 independent experiments). TM208 and Tam treatment caused a dramatic dose-dependent enhancement in the mRNA expression of EST (Figure 3A). Western blot results showed that the protein expression of EST increased significantly after TM208 and Tam treatment (Figure 3B); these results were consistent with those at the mRNA level (Figure 3). These results suggest that the regulation of EST expression by TM208 and Tam is mainly at the gene transcriptional level.

Triclosan diminished the effect of TM208 associated with the reduced survival of MCF-7 cells, and TM208 had antiestrogenic activity in vitro

The sulfation of estradiol by purified human sulfotransferases is inhibited by triclosan, which is usually used as an antibacterial agent ${ }^{[40]}$. Therefore, triclosan can be used as an inhibitor of sulfation to investigate the role of EST in the development 


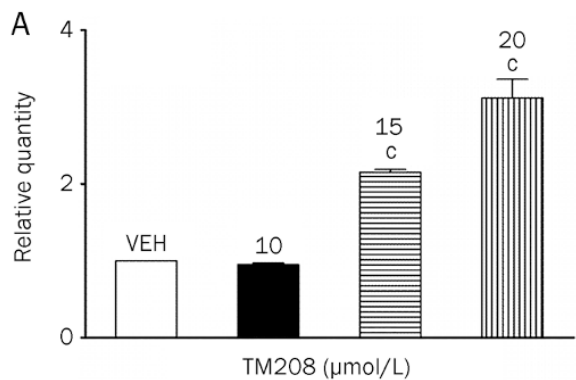

B
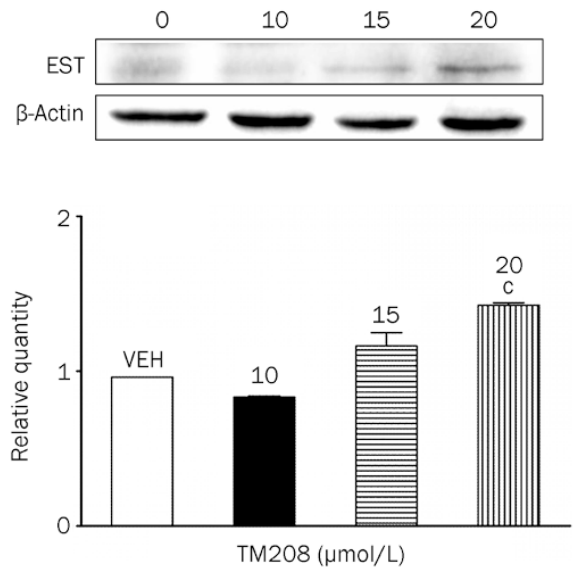
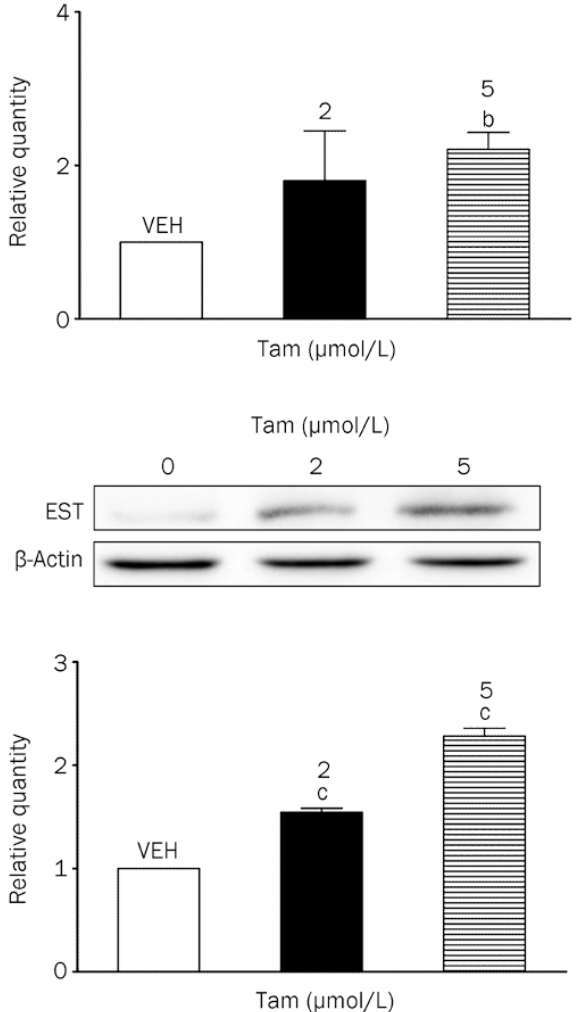

Figure 3. TM208 and Tam induced the expression of EST at the mRNA and protein levels. (A) TM208 and Tam increased the mRNA expression of EST in MCF-7 cells after $7 \mathrm{~d}$ of treatment. (B) TM208 and Tam also increased the protein expression of EST in MCF-7 cells after $7 \mathrm{~d}$ of treatment. The expression of $\beta$-actin was included as a loading control. VEH, vehicle. ${ }^{b} P<0.05,{ }^{c} P<0.01$ vs vehicle.

and progression of breast cancer. The effect of triclosan on the TM208-induced loss of cell viability was detected with SRB assays. As shown in Figure 4A, after treatment with TM208 (20 $\mathrm{\mu mol} / \mathrm{L})$ alone, the cell survival rate decreased nearly $40 \%$. When TM208 was administered in combination with triclosan, the cell survival rate increased significantly, returning to the original level of the vehicle group (Figure 4A). This result indicates that the sulfation of estrogen by EST is involved in the inhibitory effect of TM208 on the viability of MCF-7 cells.

We also investigated the effect of E2 on the survival rate of MCF-7 cells and on the antiestrogenic efficacy of TM208 using the SRB assay. Figure 4B shows that the cell survival rate of the groups treated with $0.001 \mathrm{nmol} / \mathrm{L}, 1 \mathrm{nmol} / \mathrm{L}$ and 1 $\mu \mathrm{mol} / \mathrm{L}$ of E2 increased by 2-, 2.3-, and 2.5-fold, respectively, compared with the control group, which was cultured in non-E2 media. There was no significant difference between
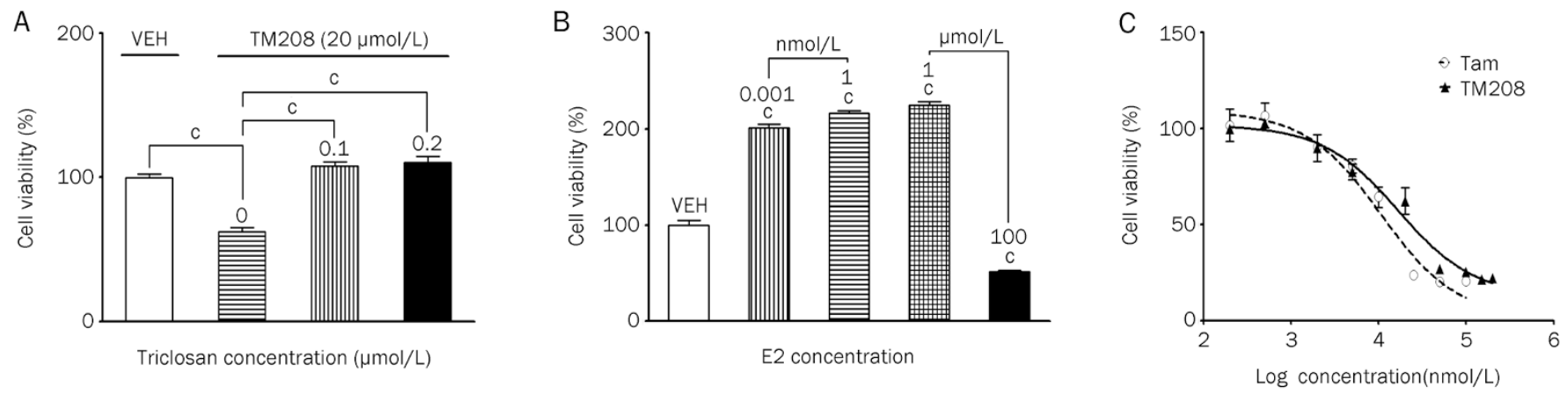

Figure 4. Triclosan diminished the effect of TM208, which was associated with the reduced survival of MCF-7 cells, and TM208 has an antiestrogenic activity in vitro. (A) The loss of viability in the MCF-7 cells treated with $20 \mu \mathrm{mol} / \mathrm{L}$ TM208 was counteracted by various doses (0.1 and $0.2 \mu \mathrm{mol} / \mathrm{L})$ of triclosan (an inhibitor of sulfation), indicating that the sulfation of estrogens by EST is involved in the anticancer efficacy of TM208. (B) The effect of E2 on the survival of MCF-7 cells. (C) TM208 and Tam exerted significant anti-estrogen effects in MCF-7 cells, as determined by the loss of cell viability. VEH, vehicle. ${ }^{c} P<0.01$ vs vehicle unless otherwise labeled. 
the three treatment groups. Conversely, $100 \mu \mathrm{mol} / \mathrm{L}$ of E2 inhibited the growth of MCF-7 cells. When MCF-7 cells were treated with $1 \mathrm{nmol} / \mathrm{L}$ of E2 in combination with TM208 or Tam, the inhibitory effect of TM208 or Tam on MCF-7 cells was observed(Figure $4 \mathrm{C}$ ), and the $\mathrm{IC}_{50}$ values of TM208 and Tam were 16.97 and $10.68 \mu \mathrm{mol} / \mathrm{L}$, respectively, which were much smaller than those observed under E2-free incubation (36.38 and $22.45 \mu \mathrm{mol} / \mathrm{L}$, respectively), suggesting that TM208 may function via a down-regulation of estrogen in cells.

TM208 and tamoxifen increased the expression and activity of EST in MCF-7 xenograft tumors

Consistent with the in vitro results, TM208 effectively induced the protein expression levels of EST in tumors in vivo (Figure 5A). The Western blot results for EST were further verified by the IHC staining results. As shown in Figure 5B, the tumors of TM208-treated mice had a much greater number of positive cells and a stronger brown staining intensity for EST expression than those in the vehicle treatment group. Although Tam and TM208 were both able to up-regulate EST expression in tumors, the effect of TM208 was remarkably stronger than that of Tam (Figure 5A, 5B).

Radioactive assay results showed that both TM208 and Tam significantly increased the enzyme activity of EST in tumors (Figure 5C). After $18 \mathrm{~d}$ of treatment with TM208 (50 and 150 $\left.\mathrm{mg} \cdot \mathrm{kg}^{-1} \cdot \mathrm{d}^{-1}\right)$ or Tam $\left(50 \mathrm{mg} \cdot \mathrm{kg}^{-1} \cdot \mathrm{d}^{-1}\right)$, the EST activity increased 2.6-, 2.7-, and 2.5-fold, respectively. In addition, real-time PCR results quantitatively revealed the time course of EST mRNA expression after multiple treatments with TM208. Compared with the vehicle treatment group, TM208 treatment significantly increased the expression of EST mRNA in tumors at each time point (Figure 5D). Moreover, as the tumors grew,
A

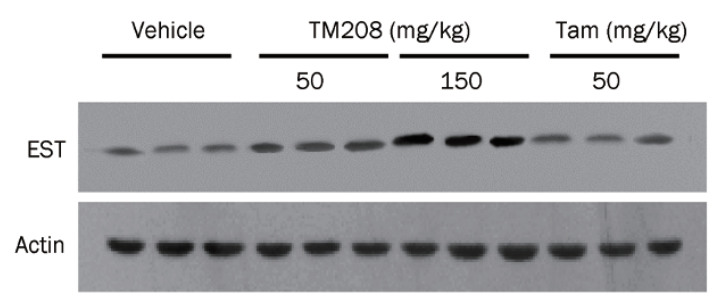

B

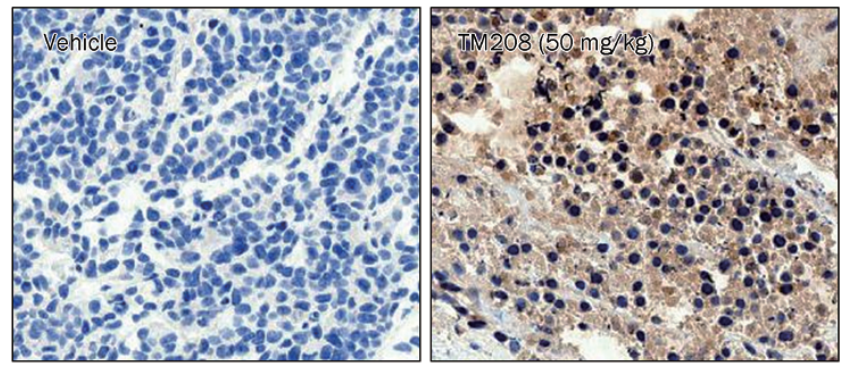

C

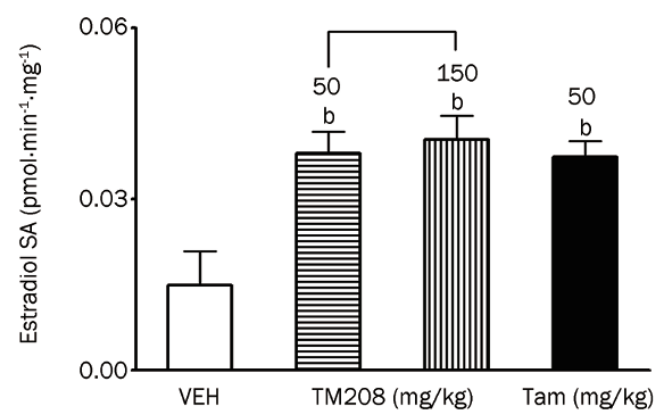

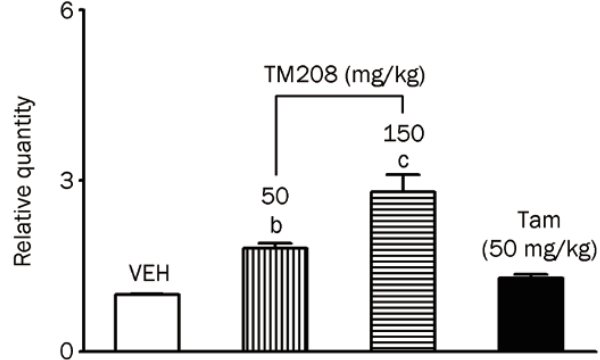
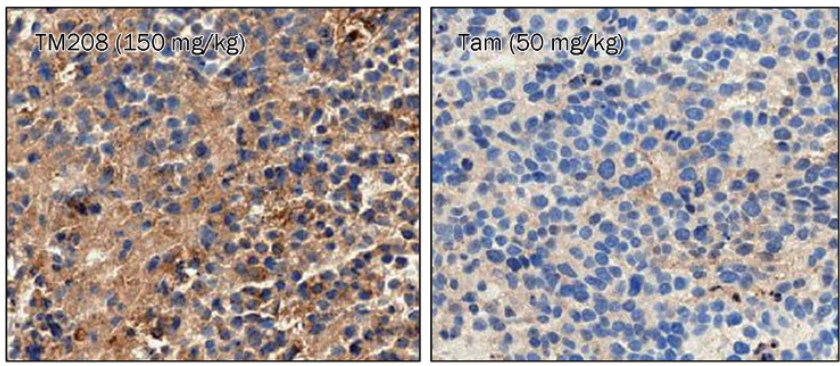

D

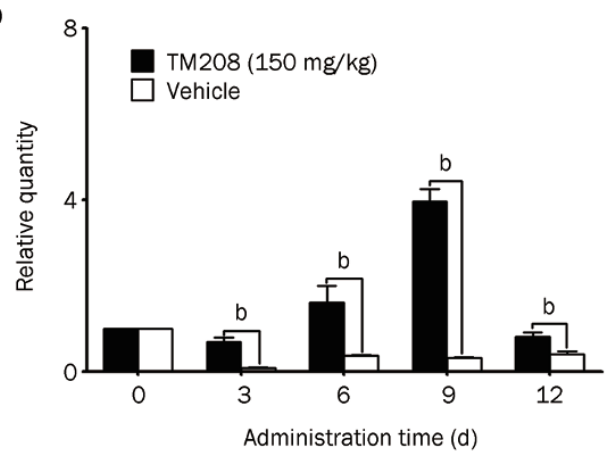

Figure 5. TM208 and Tam increased the expression and activity of EST in MCF-7 xenograft tumors. (A) Western blot results showed that TM208 and Tam induced the protein expression of intratumoral EST. The expression of $\beta$-actin was included as a loading control. (B) EST immunostaining on tumor paraffin sections also demonstrated the induction of EST by TM208 or Tam. The brown staining intensity represents the expression of EST in the cytosol of tumors, and the blue staining represents the cell nucleus. The original magnification is $100 \times$ for all panels. (C) TM208 and Tam significantly increased the enzyme activity of EST in tumors. (D) Compared with the corresponding control group at different times, the mRNA expression of EST in tumors was significantly induced by the continuous administration of TM208 (150 mg.kg $\left.\cdot \mathrm{d}^{-1}\right)$. VEH, vehicle. ${ }^{\mathrm{b}} P<0.05,{ }^{\mathrm{c}} P<0.01 \mathrm{vs}$ vehicle. 
the mRNA expression of EST in the vehicle treatment group was suppressed to a low level.

TM208 reduced the tumor estrogen level and inhibited the uterine responses

ELISA analyses quantitatively revealed the time course of the active E2 levels in tumors after treatment with TM208 (Figure $6 \mathrm{~A})$, and all E2 concentrations in the tumors were at a nanomolar level. In the vehicle treatment group, the E2 concentration increased gradually with time and plateaued on $\mathrm{d} 9$. However, after multiple doses of TM208, the E2 concentrations in tumors remained similar to those on $\mathrm{d} 0$. In addition, the uterine volume was obviously larger in tumor-bearing mice treated with vehicle than in mice treated with TM208 (Figure 6B, 6C). The typical uterine enlargement (water imbibition) stimulated by estrogens in the vehicle-treated mice was not observed in the TM208-treated mice, indicating that the level of active estrogens in the circulating system was lower in the TM208-treated mice than in the control group.

\section{Discussion}

In this study, we first investigated the anticancer efficacy of TM208. The SRB assay showed that, in vitro, under E2 or E2-free incubation, the ability of TM208 to reduce the cell viability of MCF-7 cells is weaker than that of Tam. However, the results of the tumor growth inhibition assay indicated that the mean gain in tumor volumes in groups treated with 50 $\mathrm{mg} / \mathrm{kg}$ TM208 were similar to those in groups treated with $50 \mathrm{mg} / \mathrm{kg}$ Tam, whereas the high-dose TM208 treatment (150 $\mathrm{mg} \cdot \mathrm{kg}^{-1} \cdot \mathrm{d}^{-1}$ ) caused a significant inhibition in tumor growth compared with those of the vehicle-treated controls and Tamtreated group. This result means that the anti-tumor effects of TM208 are virtually certain in MCF-7 xenograft mice, at least in the presence of physiological levels of estrogens.

In a previous study, we demonstrated that TM208 could inhibit the phosphorylation of EGFR in vitro and in vivo ${ }^{[29]}$. Considering that MCF-7 cells are both low in EGFR expression and ER-positive, we assume that the anticancer efficacy of TM208 in vivo may be due to estrogen-related mechanisms (such as EST induction) that synergize with the anti-tumor properties of TM208 and involve p-EGFR inhibition. Therefore, we tested whether the induction of EST plays a role in the anti-breast cancer effect of TM208. EST shows the highest affinity for E2 and estrone ${ }^{[15]}$, whereas other SULTs, such as SULT1A1 and SULT2A1, exhibit some affinities for E2 and estrone, which are only found at non-physiological high concentrations $^{[14,41]}$. Not counting EST, SULT1A1 was reported to play the most crucial role in the sulfation of intratumoral estrogens by its contribution to the conjugation of the metabolites of E2 (such as 4-OH-E2 and 2-methoxy-E2) metabolized by CYP1B1 and $1 \mathrm{~A} 1^{[14,42]}$. However, as for the parent levels of E2 in the tumor, all available results of previous studies have demonstrated that the intratumoral E2 concentrations were at physiological (nanomolar) levels even when expressed as $\mathrm{pg} / \mathrm{g}$ tissue or $\mathrm{pmol} / \mathrm{g}$ tissue. For example, the mean concentration of E2 in the breast tumors of premenopausal patients
A
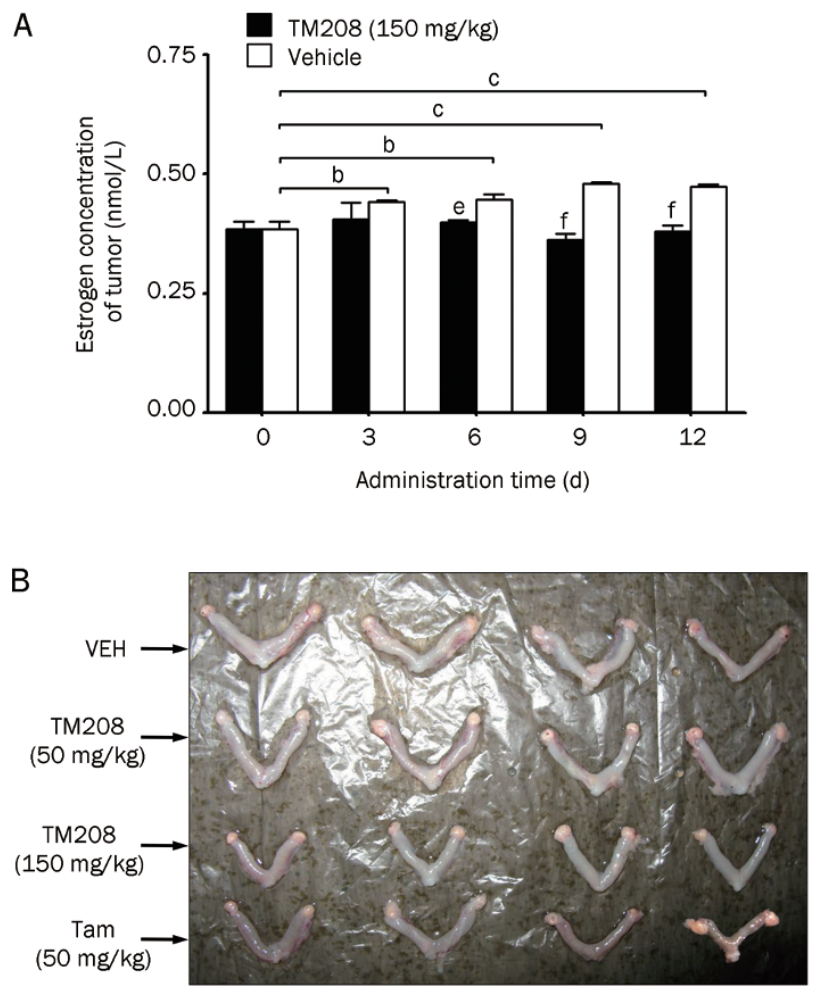

C

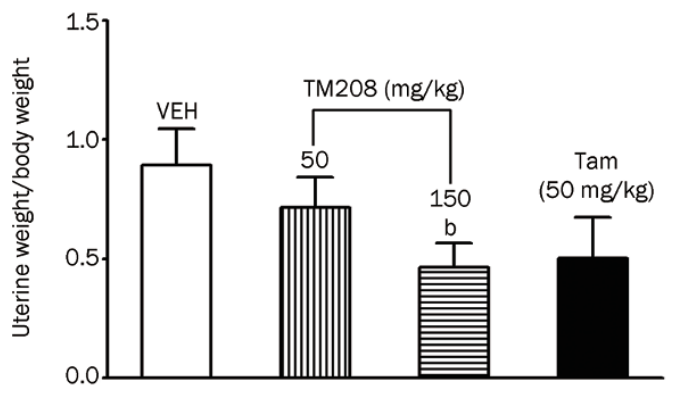

Figure 6. TM208 reduced the excessive E2 level in tumors and inhibited the uterine responses stimulated by estrogens. (A) The E2 levels in the cytosol of the tumors at each time point were much lower in the TM208treatment group than in the vehicle treatment group. ${ }^{b} P<0.05,{ }^{c} P<0.01$ vs 0 d. ${ }^{e} P<0.05,{ }^{f} P<0.01$ vs vehicle. (B) Uteri from the tumor-bearing mice and $(C)$ their weights determined with a uterotropic bioassay. $n=4$. TM208 significantly inhibited the uterine responses, as the uterine volume and weight were markedly decreased. VEH, vehicle. ${ }^{\mathrm{b}} \mathrm{P}<0.05$ vs vehicle.

was approximately $1.2 \mathrm{pmol} / \mathrm{g}$ tissue (that means that if the density of tumor is $2 \mathrm{~g} / \mathrm{mL}$, the in situ E2 concentration will be $2.4 \mathrm{nmol} / \mathrm{L})$, whereas a plasma E2 concentration of approximately $0.22 \mathrm{nmol} / \mathrm{L}$ was reported in the same paper ${ }^{[4,5]}$. Similar results were obtained from other previous studies ${ }^{[6,7]}$ and from our current study on MCF-7 xenograft tumors (0.36-0.48 $\mathrm{nmol} / \mathrm{L})$. However, we once investigated the effect of TM208 on SULT1A1 and SULT2A1 and found that TM208 induced the expression of SULT1A1 more significantly than that of SULT2A1 in both MCF-7 cells and MCF-7 xenograft tumors, 
but all of the observed increases were less than 4-fold (Figure S1-S2). However, the affinity of estrogen is much higher for EST than for SULT1A1, the $K_{\mathrm{m}}$ of which is at the nano- or micromolar level, respectively ${ }^{[15]}$. Therefore, EST is responsible for the sulfation of intratumoral activated E2, which is why we investigated EST rather than other SULT isoforms in this paper.

As the process of SULT induction is conducted on the gene transcriptional level, which is time-consuming ${ }^{[43]}$, we treated MCF-7 cells for $7 \mathrm{~d}$ to examine the effects of TM208 on EST induction in vitro. The expression of EST in the control group was at a trace level, which is in agreement with previous results $^{[42,44,45]}$. After treatment with TM208 or Tam, the mRNA and protein expression of EST increased significantly (Figure 3). Moreover, the sulfation of estrogens by EST is involved in the inhibitory effect of TM208 on the viability of MCF-7 cells with triclosan, which is a typical inhibitor of sulfation ${ }^{[40]}$. Similar to Tam, TM208 has antiestrogenic activity because it significantly diminishes the effect of E2, which is associated with the increased cell viability of MCF-7 cells (Figure 4). These results confirmed that EST induction by this compound could be used as an effective method of endocrine therapy for ERpositive breast cancer.

It has been observed in the tumors of some patients with breast cancer that EST is expressed at a low level, and this expression is inversely correlated with the breast tumor size ${ }^{[11,16]}$. Consistent with the previous results, low EST expression was observed in the absence of TM208 treatment, and this low expression increased significantly after the mice were treated with TM208 (Figure 5A). This finding was also confirmed by our immunohistochemical staining (Figure 5B) and enzyme activity (Figure 5C) results. Moreover, compared with the corresponding control group at different times, the mRNA expression of EST in tumors was significantly induced by the continuous administration of TM208 (Figure 5D). Conversely, as the tumors grew, the mRNA expression of EST in the vehicle treatment group was suppressed to a low level (Figure 5D). This result indicates that EST is inhibited with the progression of tumor growth. The observed net EST mRNA level is the sum of induction by TM208 and reduction by natural cancer disease progression. Compared with the mRNA expression of EST, as the tumors grew, the intratumoral E2 concentrations of the vehicle treatment group were $0.38 \pm 0.03,0.44 \pm 0.01,0.45 \pm 0.02,0.48 \pm 0.00$, and $0.47 \pm 0.01$ $\mathrm{nmol} / \mathrm{L}$ on $\mathrm{d} 0,3,6,9$, and $\mathrm{d} 12$; over this observation period, E2 increased by a maximum of approximately 26\% (Figure 6A). After multiple treatments with TM208, the E2 levels in the cytosol of the tumors at each of the aforementioned time points were $0.38 \pm 0.03,0.41 \pm 0.06,0.40 \pm 0.01,0.36 \pm 0.02$, and $0.38 \pm 0.02 \mathrm{nmol} / \mathrm{L}$, respectively (Figure 6A), which were much lower than those in the vehicle treatment group (except on d 0). These data are neither significantly different from each other nor from the E2 level in the normal mammary fat pad $(0.39 \pm 0.01 \mathrm{nmol} / \mathrm{L})$. The absolute reduced intratumoral E2 concentration in the TM208 treatment group is not as much as the levels observed in situ for the human breasts of healthy women versus patients ${ }^{[4,6,7]}$, but the difference was statistically significant, and all the tested concentrations were within the physiological range of E2 concentration. To the best of our knowledge, we are the first group to detect the intratumoral E2 concentration in MCF-7 xenograft mice. We found that the E2 level in tumors was significantly higher than that in normal mammary fat pads, and excessive levels of active E2 might be restored to normal levels via sulfation mediated by EST induced after the treatment with TM208, accompanied by the inhibition of tumor growth.

Pasqualini et al have demonstrated that the concentrations of estrogens in the tumors of postmenopausal patients are significantly higher than those found in the plasma, suggesting that intratumoral production of estrogens occurs ${ }^{[4]}$. In the current study, we tried to determine the free E2 plasma concentration using the same ELISA kit, but the concentration was too low to be detectable, indicating the plasma E2 concentration is much lower, which is in agreement with the previous results. Additionally, because stronger uterine estrogen responses are indicative of higher levels of active estrogens and vice versa $^{[17,46]}$, uterine estrogen responses are usually used to indirectly evaluate the overall level of active estrogens in the body. In this study, TM208 significantly inhibited the uterine responses because the uterine volume and weight obviously decreased after treatment with TM208 (Figure 6B), indicating that TM208 reduced the levels of circulating estrogen. These results suggest that TM208 may exert its anti-tumor effect via regulating both the intratumoral and circulating E2 concentration as well as inhibiting the EGFR-signaling pathway ${ }^{[29]}$.

Our study is the first to show that Tam is also a typical EST inducer. Similar to TM208, Tam remarkably induced mRNA expression and increased the enzyme activity of EST in tumors (Figures 3 and 5). Although Tam has been well known as an antagonist of the estrogen receptor in breast tissue via its active metabolites (ie, 4-hydroxy tamoxifen and N-desmethyl4-hydroxytamoxifen) ${ }^{[37]}$, our results support that part of its anti-estrogen effect may be due to the inactivation of active estrogen by sulfation via the induction of SULTs, which may cause another type of ER antagonism with Tam. Interestingly, several years ago, Tam was found to significantly induce rSULT2A1 and rSULT1A1 in rat livers and intestines ${ }^{[20]}$, which is in agreement with our present results in tumors.

The regulation of the cytosolic sulfotransferases (including EST) by nuclear receptors, such as LXR, AhR, PXR, CAR, PPAR, and Nrf2, has been well summarized ${ }^{[47,48]}$. Gong et al reported that the activation of EST is mediated by both the glucocorticoid receptor (GR) and orphan nuclear receptor liver $X$ receptor (LXR), leading to estrogen deprivation and the inhibition of breast tumor growth in vivo ${ }^{[17,46]}$. Furthermore, these responses were not observed in EST knockout mice after treatment with LXR agonists. Moreover, LXR has been shown to promote apoptosis and repress estrogen ${ }^{[46]}$, which may contribute to the anti-breast cancer efficacy of TM208. Because of the important role of LXR, we preliminarily investigated the effect of TM208 on LXR in tumors. Our results revealed that TM208 effectively induced the expression of LXR- $\alpha$ and 
LXR- $\beta$ in tumors. In addition, Tam exhibited an inducing efficacy, which was similar to the effect of TM208 on LXR in tumors (Figure S3). However, more investigation is required to determine whether SULT expression is activated directly by LXR. In addition, further experiments are needed to confirm whether GR and other nuclear receptors are involved in the underlying mechanisms of TM208-mediated EST induction.

In summary, EST induction and, accordingly, the downregulation of estradiol by TM208 are involved in anti-breast cancer efficacy. With its good pharmacokinetic characteristics, very low toxicity, and additional EGFR-pathway inhibition, TM208 is a promising candidate compound for the treatment and prevention of ER-positive breast cancers.

\section{Abbreviations}

TM208, 4-methyl-piperazine-1-carbodithioc acid 3-cyano3,3-diphenylpropyl ester hydrochloride; SULT, sulfotransferase; EST, estrogen sulfotransferase; Tam, Tamoxifen; E2, $17 \beta$-estradiol; ER, estrogen receptor; and PAPS, 3'-phosphoadenosine- $5^{\prime}$-phosphosulfate.

\section{Acknowledgements}

This work was supported by the National Natural Science Foundation of China (NSFC) (Grant 81273583).

We thank Prof Run-tao LI (School of Pharmaceutical Sciences, Peking University) for kindly providing the TM208 compound. We are also thankful to Dr David RINGER (American Cancer Society) for kindly providing the EST antibody.

\section{Supplementary information}

Supplementary figures are available at the Acta Pharmacologica Sinica website.

\section{Author contribution}

Tian-yan ZHOU, Xi-wei JI, and Guang-ping CHEN designed the research; Xi-wei JI, Ming HUA, Li-jie WANG, Si-yuan WANG, Liang LI, Yan SONG, and Yin YUAN performed the research; Xi-wei JI, Tian-yan ZHOU, and Wei LU analyzed the data; Xi-wei JI and Tian-yan ZHOU wrote the manuscript.

\section{References}

1 Jensen EV. From chemical warfare to breast cancermanagement. Nat Med 2004; 10: 1018-21.

2 Yager JD, Davidson NE. Estrogen carcinogenesis in breast cancer. N Engl J Med 2006; 354: 270-82.

3 Yaghjyan L, Colditz GA. Estrogens in the breast tissue: a systematic review. Cancer Causes Control 2011; 22: 529-40.

4 Pasqualini JR, Chetrite G, Blacker C, Feinstein MC, Delalonde L, Talbi $\mathrm{M}$, et al. Concentrations of estrone, estradiol, and estrone sulfate and evaluation of sulfatase and aromatase activities in pre- and postmenopausal breast cancer patients. J Clin Endocrinol Metab 1996; 81: 1460-4.

5 Pasqualini JR. The selective estrogen enzyme modulators in breast cancer: a review. Biochim Biophys Acta 2004; 1654: 123-43.

6 Chetrite GS, Cortes-Prieto J, Philippe JC, Wright F, Pasqualini JR. Comparison of estrogen concentrations, estrone sulfatase and aromatase activities in normal, and in cancerous, human breast tissues. J Steroid Biochem Mol Biol 2000; 72: 23-7.

7 Shibuya R, Suzuki T, Miki Y, Yoshida K, Moriya T, Ono K, et al. Intratumoral concentration of sex steroids and expression of sex steroidproducing enzymes in ductal carcinoma in situ of human breast. Endocr Relat Cancer 2008; 15: 113-24.

8 Pasqualini JR, Chetrite GS. Recent insight on the control of enzymes involved in estrogen formation and transformation in human breast cancer. J Steroid Biochem Mol Biol 2005; 93: 221-36.

9 Suzuki T, Miki Y, Nakamura Y, Moriya T, Ito K, Ohuchi N, et al. Sex steroid-producing enzymes in human breast cancer. Endocr Relat Cancer 2005; 12: 701-20.

10 Suzuki T, Miki Y, Moriya T, Akahira J, Hirakawa $\mathrm{H}$, Ohuchi $\mathrm{N}$, et al. In situ production of sex steroids in human breast carcinoma. Med Mol Morphol 2007; 40: 121-7.

11 Suzuki T, Nakata T, Miki Y, Kaneko C, Moriya T, Ishida T, et al. Estrogen sulfotransferase and steroid sulfatase in human breast carcinoma. Cancer Res 2003; 63: 2762-70.

12 Jordan VC. Tamoxifen as the first targeted long-term adjuvant therapy for breast cancer. Endocr Relat Cancer 2014; 21: R235-46.

13 Mouridsen H, Giobbie-Hurder A, Goldhirsch A, Thurlimann B, Paridaens R, Smith I, et al. Letrozole therapy alone or in sequence with tamoxifen in women with breast cancer. N Engl J Med 2009; 361: 766-76.

14 Fu J, Weise AM, Falany JL, Falany CN, Thibodeau BJ, Miller FR, et al. Expression of estrogenicity genes in a lineage cell culture model of human breast cancer progression. Breast Cancer Res Treat 2010; 120: $35-45$.

15 Zhang H, Varlamova O, Vargas FM, Falany CN, Leyh TS. Sulfuryl transfer: the catalytic mechanism of human estrogen sulfotransferase. J Biol Chem 1998; 273: 10888-92.

16 Sasano H, Suzuki T, Nakata T, Moriya T. New development in intracrinology of breast carcinoma. Breast Cancer 2006; 13: 129-36.

17 Gong H, Jarzynka MJ, Cole TJ, Lee JH, Wada T, Zhang B, et al. Glucocorticoids antagonize estrogens by glucocorticoid receptor-mediated activation of estrogen sulfotransferase. Cancer Res 2008; 68: 738693.

18 Xu Y, Liu X, Guo F, Ning Y, Zhi X, Wang X, et al. Effect of estrogen sulfation by SULT1E1 and PAPSS on the development of estrogendependent cancers. Cancer Sci 2012; 103: 1000-9.

19 Pasqualini JR, Chetrite GS. Correlation of estrogen sulfotransferase activity and proliferation in normal and carcinomatous human breast. A hypothesis. Anticancer Res 2007; 27: 3219-25.

20 Maiti S, Chen G. Tamoxifen induction of aryl sulfotransferase and hydroxysteroid sulfotransferase in male and female rat liver and intestine. Drug Metab Dispos 2003; 31: 637-44.

21 Chen X, Baker SM, Chen G. Methotrexate induction of human sulfotransferases in HepG2 and Caco-2 cells. J Appl Toxicol 2005; 25: 354-60.

22 Chen Y, Huang C, Zhou T, Zhang S, Chen G. Biochanin A induction of sulfotransferases in rats. J Biochem Mol Toxicol 2010; 24: 102-14.

23 Ge ZM, Li RT, Cheng TM, Cui JR. Synthesis and biological activities of diquaternary dipiperazinium salts containing dithiocarboxyl groups. Arch Pharm (Weinheim) 2001; 334: 173-6.

24 Guo BG, Ge ZM, Cheng TM, Li RT. Synthesis and anti-tumor activities of 1,4-bis[3-(amino-dithiocarboxy)propionyl]piperazine derivatives. Yao Xue Xue Bao 2001; 36: 185-7.

25 Li RT, Cheng TM, Cui JR. Study on the synthesis and anticancer activity of dithiocarbamate. US Pat: 1328999, 2002-01 2002-01.

26 Li RT, Cheng TM, Cui JR. Piperazine mono(dithio)-carbamate ester compounds and analog: preparation method and pharmaceutical 
use. P.US Pat: 10/157733 2002-05; WO Apply No: PCT/US02/16772 2002-05 2002-05.

27 Zhang N, Guo W, Wang L, Huang W, Xu B, Ge Z, et al. Effect of TM208 on QGY-7703 xenograft tumor growth. Anticancer Drugs 2008; 19 : 593-8.

28 Guo W, Ran FX, Wang RQ, Cui JR, Li RT, Cheng TM, et al. Antitumour effect of hydrochloride 4-methyl-piperazine-1-carbodithioc acid 3-cyano-3,3-diphenyl-propyl ester. Chin J Clin Pharmacol Ther 2004; 9: $59-62$.

29 Ji XW, Li RT, Li ZQ, Li L, Shao XY, Wang SY, et al. Inhibition of EGFR autophosphorylation plays an important role in the anti-breast cancer efficacy of the dithiocarbamate derivative TM208. Acta Pharmacol Sin 2014; 35: 239-47.

30 Yarden RI, Wilson MA, Chrysogelos SA. Estrogen suppression of EGFR expression in breast cancer cells: a possible mechanism to modulate growth. J Cell Biochem Suppl 2001; Suppl 36: 232-46.

31 Jiang X, Ling X, Han F, Li R, Cui J. Studies on the metabolism of 4-methyl-piperazine-1-carbodithioc acid 3-cyano-3,3-diphenylpropyl ester hydrochloride in rats by high-performance liquid chromatography/electrospray ionization tandem mass spectrometry. J Pharm Biomed Anal 2007; 44: 1127-32.

32 Maiti S, Zhang J, Chen G. Redox regulation of human estrogen sulfotransferase (hSULT1E1). Biochem Pharmacol 2007; 73: 147481.

33 Falany CN, Krasnykh V, Falany JL. Bacterial expression and characterization of a cDNA for human liver estrogen sulfotransferase. J Steroid Biochem Mol Biol 1995; 52: 529-39.

34 Vichai V, Kirtikara K. Sulforhodamine B colorimetric assay for cytotoxicity screening. Nat Protoc 2006; 1: 1112-6.

35 Ahn KH, Yi KW, Park HT, Shin JH, Hur JY, Kim SH, et al. Anti-proliferative effect of Klimaktoplan(R) on human breast cancer cells. Arch Gynecol Obstet 2013; 288: 833-8.

36 Zhou T, Huang C, Chen Y, Xu J, Shanbhag PD, Chen G. Methamphetamine regulation of sulfotransferase $1 \mathrm{~A} 1$ and $2 \mathrm{~A} 1$ expression in rat brain sections. Neurotoxicology 2013; 34: 212-8.

37 Johnson MD, Zuo H, Lee KH, Trebley JP, Rae JM, Weatherman RV, et al. Pharmacological characterization of 4-hydroxy-N-desmethyl tamoxifen, a novel active metabolite of tamoxifen. Breast Cancer Res Treat 2004; 85: 151-9.

38 Huang $\mathrm{C}$, Chen $\mathrm{Y}$, Zhou T, Chen G. Sulfation of dietary flavonoids by human sulfotransferases. Xenobiotica 2009; 39: 312-22.

39 Joyner A, Wall N. Immunohistochemistry of whole-mount mouse embryos. Cold Spring Harbor Protocols 2008; doi:10.1101/pdb. prot4820.

40 Wang LQ, Falany CN, James MO. Triclosan as a substrate and inhibitor of 3'-phosphoadenosine 5'-phosphosulfate-sulfotransferase and UDPglucuronosyl transferase in human liver fractions. Drug Metab Dispos 2004; 32: 1162-9.

41 Gamage N, Barnett A, Hempel N, Duggleby RG, Windmill KF, Martin JL, et al. Human sulfotransferases and their role in chemical metabolism. Toxicol Sci 2006; 90: 5-22.

42 Spink BC, Katz BH, Hussain MM, Pang S, Connor SP, Aldous KM, et al. SULT1A1 catalyzes 2-methoxyestradiol sulfonation in MCF-7 breast cancer cells. Carcinogenesis 2000; 21: 1947-57.

43 Aranda A, Pascual A. Nuclear hormone receptors and gene expression. Physiol Rev 2001; 81: 1269-304.

44 Falany JL, Falany CN. Expression of cytosolic sulfotransferases in normal mammary epithelial cells and breast cancer cell lines. Cancer Res 1996; 56: 1551-5.

45 Smuc T, Rizner TL. Expression of 17beta-hydroxysteroid dehydrogenases and other estrogen-metabolizing enzymes in different cancer cell lines. Chem Biol Interact 2009; 178: 228-33.

46 Gong H, Guo P, Zhai Y, Zhou J, Uppal H, Jarzynka MJ, et al. Estrogen deprivation and inhibition of breast cancer growth in vivo through activation of the orphan nuclear receptor liver $\mathrm{X}$ receptor. Mol Endocrinol 2007; 21: 1781-90.

47 Alnouti Y, Klaassen CD. Regulation of sulfotransferase enzymes by prototypical microsomal enzyme inducers in mice. J Pharmacol Exp Ther 2008; 324: 612-21.

48 Runge-Morris M, Kocarek TA, Falany CN. Regulation of the cytosolic sulfotransferases by nuclear receptors. Drug Metab Rev 2013; 45: 15-33. 